\title{
LA MARGINALIDAD ELECTORAL Y POLÍTICA DE LA EXTREMA DERECHA VALENCIANA (2003-2015)
}

\author{
Electoral and political marginalization of the \\ extreme right valencian (2003-2015)
}

\author{
ANA López ORTEGA ${ }^{1}$ \\ Universitat de València \\ Valencia, España \\ alor5@uv.es
}

Vol. XV, N²6, 2017, 67-92

Recepción: 9 de marzo de 2017

Aceptación: 8 de junio de 2017

Versión final: 28 de julio de 2017

RESUMEN. El partido político España 2000 se auto define como «social patriota» y «anti inmigración» y en su discurso se aprecian elementos de formaciones neopopulistas europeas que han obtenido importantes resultados electorales en la última década. Sin embargo, España 2000, tras más de diez años de historia, no ha conseguido el éxito de sus homólogos europeos. Por ello, el texto trata de ofrecer las claves que han favorecido su emergencia y moderado crecimiento en esta región española a través de una metodología cuantitativa y la técnica de inferencia ecológica y a partir de sus cifras electorales. El resultado muestra el posible origen de su electorado y las características sociodemográficas de sus votantes. Finalmente se reflexiona sobre el futuro político de la formación y de la extrema derecha española, que actualmente ocupa una posición marginal en el sistema político.

Palabras clave: partido político, extrema derecha, ideología, elecciones, discurso

ABSTRACT. The political party España 2000 was self defined as «social-patriot» and "anti-immigration» and in his speech are appreciated elements of European populist neo formations that

\footnotetext{
Doctora en Ciencias Políticas. Investigadora de la Generalitat Valenciana.
} 
have obtained important election results over the last decade. However, España 2000, after more than ten years of history, has failed the success of his European counterparts. Therefore, the text seeks to provide the keys that have favored its emergency and moderate growth in this Spanish region through a quantitative methodology and technique of ecological inference and from their electoral numbers. The result shows the possible origin of their electorate and the socio-demographic characteristics of their voters. Finally, it reflects on the political future of the formation and the Spanish extreme right, which currently occupies a marginal position in the political system.

Keywords: political party, extreme right, ideology, elections, speech

\section{INTRODUCCIÓN}

El partido político patriota y anti inmigración España 2000, constituido en 2003 en la ciudad de Valencia, se convirtió en 2007 en el principal partido del espacio político de extrema derecha en la Comunitat Valenciana y consiguió aproximadamente 6.000 votos. Cuatro años más tarde, duplicó estos resultados y consiguió 4 representantes locales. Asimismo, la formación ha aumentado el número de municipios donde han presentado candidaturas en las citas locales desde 2003 hasta 2015.

Además, el partido ha logrado una mayor presencia en los medios de comunicación tradicionales y ha mejorado la calidad de sus publicaciones en internet, dotándolas de una mayor sofisticación y profesionalización en el contenido y la imagen, junto con una actualización diaria de sus redes sociales. Igualmente, España 2000 ha desarrollado su actividad «a pie de calle», es decir, en el ámbito municipal, a través de manifestaciones y actos protesta. Una estrategia que ha combinado con otras formas de movilización similares a las de otras formaciones europeas que han experimentado un gran crecimiento electoral (Amanecer Dorado, Frente Nacional, Partido por la Libertad austríaco) como el reparto de alimentos «solo para nacionales».

En este marco, el objetivo principal del artículo es analizar la evolución electoral y política del partido político España 2000 y comprender las claves del apoyo electoral recibido. Igualmente, detectar aquellas debilidades en el proyecto político que sitúan a la formación en un segundo plano en la escena política valenciana.

Partimos de la hipótesis de que el partido ha desarrollado un discurso similar al de otras formaciones neopopulistas europeas, especialmente en cuestiones como la inmigración y su concepción (autoritaria y res- 
trictiva) del Estado, y que además se visibiliza a partir de las elecciones autonómicas de 2007. Entre otros motivos, para intentar distanciarse del estigma de fascista e incluso neonazi que los medios de comunicación le han otorgado desde su constitución en 2003. Sin embargo, con este nuevo discurso la formación ha logrado obtener unos resultados electorales discretos, que han aumentado ligeramente en localidades y comarcas durante tres elecciones consecutivas, pero poco significativos en el conjunto de esta comunidad autónoma.

Por este motivo, a través de su discurso y análisis electoral, intentaremos comprender qué factores han propiciado su aparición y su moderado aumento en la Comunitat Valenciana, ya que «el primer paso para entender a la derecha radical como fenómeno político es explicar su aparición» (De Lange, 2007: 112).

Para ello, el artículo se estructura en cuatro grandes apartados. En primer lugar, y a modo de introducción del tema, la investigación estudia los motivos de los recientes triunfos electorales de los partidos políticos calificados de extrema derecha en la Unión Europea en la última década. Posteriormente se revisan los principales argumentos que explican la situación de excepcionalidad española, donde no han emergido formaciones de extrema derecha como en el resto de Europa.

A continuación se analizan las principales características del partido político España 2000: estructura, actividad política y, en un tercer apartado, se profundiza en su evolución electoral (2003-2015), como punto de partida para intentar explicar los motivos de la emergencia de una formación con un discurso neopopulista en la Comunitat Valenciana.

En una tercera parte y debido a la actual inexistencia de estudios sobre el partido, se elabora una descripción del posible perfil del votante mediante la comparación de diferentes variables (sociodemográficas, socioeconómicas y políticas) y la posterior recodificación en el eje político 9-10, correspondiente a la extrema derecha, y extraídas de la pregunta de auto posicionamiento de las encuestas postelectorales de las elecciones autonómicas del CIS de 2007, 2011, 2013 y 2015.

Después de este análisis, se proponen los factores que podrían explicar la emergencia de la formación valenciana en la Comunitat Valenciana a partir de las teorías que plantean una "estructura de oportunidades políticas" (Arzheimer, 2009) necesarias para el crecimiento de este tipo de partidos políticos y que analizan factores como la inmigración, la tasa de parto o el diseño institucional.

Finalmente se exponen las principales ideas del artículo y se reflexiona sobre las principales fortalezas y debilidades del proyecto polí- 
tico inserto en el ámbito autonómico y también como un futuro sujeto político-nacional.

La metodología de estudio que se ha empleado es mixta: aunque prevalece la de tipo cualitativo, también se ha recurrido a la cuantitativa (datos electorales y encuestas) con la finalidad de analizar de la manera más completa, rigurosa y precisa el complejo objeto de estudio.

Respecto a las fuentes de información que se utilizan en el texto, se han combinado las fuentes secundarias y de trabajo propia de la autora. En la primera parte del artículo se ha recurrido a las investigaciones académicas de la familia de la derecha radical populista con el objetivo de determinar las características, el discurso, el electorado y la militancia del partido. En segundo lugar, se han utilizado los resultados electorales de las elecciones autonómicas y municipales desde 2003 hasta 2015 en la Comunitat Valenciana. También las encuestas de opinión postelectorales del CIS 2003-2015.

\section{El avance electoral de la extrema derecha europea}

El estudio que desarrollamos parte del contexto europeo donde se ha confirmado el avance electoral e incluso una alianza de partidos calificados de «nueva extrema derecha» (Ignazi, 2003) o «derecha radical populista» (Mudde, 2007) en prácticamente todos los parlamentos nacionales de la Unión Europea. Taguieff (2007) ha llegado a calificar este ascenso como de «ola populista». Rydren (2007) sostiene que la exitosa movilización política de estos partidos se ha construido a partir de un «programa etno-nacionalista basado en el racismo cultural y una retórica populista (pero no antidemocrática) anti-establishment político».

Este crecimiento confirma que la extrema derecha ya no es una moda pasajera, sobre todo tras los resultados obtenidos por algunas de estas formaciones en las elecciones al Parlamento Europea en mayo de 2015, donde 76 diputados de partidos de extrema derecha consiguieron por primera vez un alto porcentaje de representación con un discurso eurófobo, ultra nacionalista y anti-inmigrante. Pero también la victoria del Brexit en el Reino Unido, que ha establecido un precedente en la Unión Europea al decidir un Estado en referéndum abandonar el espacio europeo, supone el triunfo de uno de los objetivos prioritarios de los partidos de esta familia ideológica: la desintegración del proyecto europeo. Además, la victoria del republicano Donald Trump en los comicios presidenciales de Estados Unidos, en noviembre de 2016 - con un programa ultra proteccionista y xenófobo en contra de los inmigrantes, tanto 
latinos como musulmanes-, lo ha convertido en un modelo a imitar para el resto de líderes europeos populistas.

Además el crecimiento electoral de estas formaciones ha venido acompañado de la capacidad de influencia de la derecha radical en el establecimiento de la agenda política europea, a través de un discurso articulado y unificador, que impregna las prácticas de los grandes partidos conservadores y socialdemócratas europeos con prácticas que provocan el retroceso en los estándares democráticos. El último ejemplo de esta influencia en la política de la Unión Europea se materializa en las políticas de integración y de acogida de los refugiados y la aprobación por el Consejo Europeo el 19 de marzo de 2016 del denominado Tratado de la Vergüenza (el acuerdo entre Unión Europea y Turquía sobre la devolución automática y masiva de los extranjeros llegados a las costas de Grecia desde Turquía, incluidos los refugiados sirios).

En cambio, España es uno de los países europeos con menor presencia de partidos y formaciones de extrema derecha y no se ha generado un proceso de modernización en masa de estos partidos. La extrema derecha «no capitaliza esta tormenta perfecta como otros países europeos» (Casals Meseguer, 2011:35). El primer factor que explicaría esta situación calificada de "excepcional" en Europa es que en España la mayoría de estos partidos han permanecido anclados en programas y discursos vinculados al pasado dictatorial (Falange Auténtica, Falange Española de las Jons, Alianza Nacional). También la falta de líderes carismáticos y su incapacidad de aunar esfuerzos ha impedido vertebrar un gran partido estatal que rentabilizara en las urnas la crisis actual. Al mismo tiempo, Casals sostiene que la presencia hegemónica del Partido Popular (PP) en este espacio electoral que capitaliza todos los votos de la derecha impide el éxito de partidos ubicados en el continuum ideológico de la extrema derecha. Alonso y Rovira (2015) argumentan que es la tradición bipartidista española la que hace prácticamente imposible que los partidos minoritarios puedan obtener un número significativo de votos en el ámbito nacional.

Sin embargo, el primer y único caso relevante hasta el momento en España de estas formaciones ha sido el de Plataforma per Catalunya. En noviembre de 2010, la formación catalana obtuvo 75.000 votos en las elecciones autonómicas. El partido fue comparado con partidos con gran éxito electoral como el Frente Nacional francés o el Partido por la Libertad de Austria. En este sentido, Plataforma per Catalunya se considera como el primer partido de derecha radical populista en España (Hernández Carr, 2012). Sin embargo, su ciclo electoral acabó en las elecciones de 2014 con la pérdida de liderazgo de Josep Anglada del partido. 
Pese a estos buenos resultados, Plataforma per Catalunya no ha logrado a lo largo de su historia irrumpir en la política nacional como otras formaciones europeas. No obstante, su influencia sí que ha supuesto la emergencia de partidos que han tratado de imitar la "fórmula del éxito» desarrollada por Plataforma per Catalunya y algunas de sus fortalezas, como la fuerte implantación en municipios con mucha población extranjera y alto índice de paro, un discurso basado en el rechazo de la población extranjera y la crítica hacia los partidos políticos tradicionales (Hernández Carr, 2012). En ese sentido, este artículo plantea la posibilidad de que se haya producido un "efecto contagio" de algunos de los elementos de la «fórmula de éxito» desarrollada por Plataforma per Catalunya en el partido político España 2000, concretamente de su estrategia de movilización municipal.

\section{ESPAÑA 2000, LA EMERGENCIA DE UN NUEVO SUJETO POLÍTICO en la Comunitat Valenciana}

España 2000 se constituye oficialmente como partido político en 2003 en la ciudad de Valencia. En las bases de constitución del partido, publicadas en su web (www.espana2000.org), se especifica la adopción de un mecanismo de democracia interna para la elección de su presidente y de la junta directiva integrada por 26 vocales. Se trata de una organización jerárquica y piramidal donde su presidente, José Luis Roberto, está en la cúspide y en un segundo escalón los vocales. En un nivel inferior, se ubican las juntas, que se distribuyen de acuerdo a un criterio territorial y son de dos tipos: juntas provinciales (Valencia, Alicante y Castellón) y locales (29 en la ciudad de Valencia, 7 en Alicante y 5 en Castellón).

En la parte inferior de la pirámide y desde el ámbito local, el partido se organiza en las milicias, escuadras y centurias que se constituyen por dos motivos: el número de personas y las afinidades lúdico-deportivas y religiosas. Respecto al número de militantes del partido, la formación tenía poco más de 1.000 afiliados en 2003 en la Comunitat Valenciana y en 2016 creció hasta los 3.300 aproximadamente ${ }^{2}$.

2 Esta militancia se subdivide en tres tipos de miembros: los «militantes» que pagan una cuota mensual de 10 euros, los «militantes-simpatizantes» que están exentos de estas cuotas por su peso político o histórico y los «simpatizantes», que no pagan pero tienen carnet y participan en actividades. Esta información se ha extraído de las entrevistas realizadas al presidente por la autora a lo largo de la investigación. 
El fundador y líder del partido, José Luis Roberto, es un empresario y abogado vinculado a la ultraderecha y a empresas de prostitución, deportes y seguridad. Estas conexiones han provocado que Roberto fuera investigado desde los medios de comunicación, especialmente cuando era Secretario General Técnico de la Asociación Nacional de Locales de Alterne (Anela) por organizar campeonatos de boxeo y empresas de comercialización de ropa militar en Madrid.

Como máximo responsable de la formación, José Luis Roberto ejerce un fuerte control del partido con una presencia indiscutible en cada acción política, mediática y económica. Es precisamente el dueño de empresas de seguridad, academias y gimnasios donde sus trabajadores o clientes son también militantes de España 2000. Desde la perspectiva más política, José Luis Roberto lideró Plataforma España 2000 en el año 2000 y concurrió en las elecciones generales por primera vez ese mismo año. Esta plataforma, en la que estaban integradas las formaciones Movimiento Social Republicano, Democracia Nacional y Vértice, obtuvo 1.516 votos $(0,06 \%)$ y se disolvió dos años más tarde. Durante la presidencia al frente de España 2000, Roberto ha sido denunciado por diversas organizaciones, como SOS Racismo, por incitar al odio racial, la violencia y la discriminación (Informe Raxen, 2015).

Junto a la figura del líder, destaca la figura de Ernesto Milà, miembro permanente de la ejecutiva y responsable del proyecto político, propaganda e ideología desde 2007 cuando inició su carrera política en la organización neonazi PENS (Partido Español Nacional Socialista). La entrada de Milà constituye un factor decisivo para la transformación del discurso del partido y su homologación al adoptado por otros partidos neopopulistas europeos. En este sentido, este nuevo miembro ha reiterado que el partido «debe ganar legitimidad» (España 2000, 2011), y para ello, debe distinguirse de la estigmatización de la extrema derecha española con un discurso más europeo.

El examen de la trayectoria política del partido muestra dos etapas. En ellas se observa un cambio de las principales temáticas de movilización y de sus actividades políticas. La primera comenzaría en 2003 y se extendería hasta 2007, aproximadamente (prcampaña electoral autonómica). En esta primera época, el partido opta por la exaltación de la unidad española y el anti catalanismo ${ }^{3}$. Además, su actividad política se

El partido boicoteó la presentación del libro del sociólogo Vicent Flor, Noves Glòries a Espanya, sobre el anticatalanismo en la Comunitat Valenciana. que atribuía a España 2000 la difusión de mensajes como "catalanistas terroristas". Más información en http://www.elmundo.es/elmundo/2011/07/05/valencia/1309891348.html 
centró en manifestaciones puntuales sobre estos dos temas -el Día de la Comunitat Valenciana (9 de octubre) y el Día de la Hispanidad (12 de octubre). Durante esta etapa, el partido fue protagonista en reportajes televisivos y publicaciones sobre su presidente y su vinculación con neonazis, empresas de prostitución, seguridad, gimnasios y peleas ilegales (Salas, 2011).

En cambio, a partir de las elecciones de 2007, el discurso del partido se concentra en el eje "autóctono-extranjero» que se visibiliza en la adopción de un programa anti inmigración y ultra conservador diferenciado del partido conservador mayoritario ${ }^{4}$, el Partido Popular (PP). Precisamente en la Comunitat Valenciana, el PP ha adoptado desde 2008 unas políticas de inmigración de carácter integrador, que se implantan oficialmente a partir de la aprobación de la Ley 15/2008 de Integración de las Personas Inmigrantes en la Comunitat Valenciana ${ }^{5}$, del 5 de diciembre. Esta ley y las actuaciones previas del gobierno Popular se convirtieron en objeto de crítica en las campañas electorales de España 2000 en 2007, 2011 y 2015. Además, en 2010 presentaron mociones para que no se empadronaran inmigrantes ilegales en los municipios donde la formación tenía representación (Silla, Onda, Dos Aguas). Conjuntamente desarrollaron una campaña por varios plenos municipales contra el empadronamiento de inmigrantes ilegales (Silla, Onda, Torrent, Paiporta, Godella).

Al mismo tiempo, España 2000 ha intentado desvincularse de su estigma violento y ha adoptado medidas como prohibir la exhibición de símbolos fascistas en manifestaciones públicas o cualquier tipo de exaltación al franquismo. Igualmente, la formación aprobó un documento político en 2007 donde se definió como un "partido nuevo, demócrata y que en ningún caso pretende acabar con el sistema, sino que aspira a convertirse en una alternativa real a los partidos políticos tradicionales, a los que acusa de corruptos y de ser la causa del declive social, político y económico que ha surgido con la crisis económica de 2007» (España 2000, 2007).

Por otro lado, en los programas electorales de 2011 y 2015 es posible identificar tres variables centrales que se han considerado comunes

4 España 2000 incluye en sus campañas mensajes como: «La inmigración supone una invasión pacífica del territorio nacional y por ello hay que reformar el Código Civil para que sea más difícil obtener la nacionalidad» (España 2000, 2007). «El derecho de voto de los ciudadanos extranjeros debe ser revocado y hay que reducir las prestaciones sociales a los inmigrantes» (España 2000, 2011).

5 Esta Ley aprobó programas de inserción socio-laboral y cultural y otras medidas que favorecían la integración de personas inmigrantes en la Comunitat Valenciana. 
a la familia de partidos de la derecha radical populista: nacionalismo, populismo y autoritarismo (Mudde, 2007). En este sentido, España 2000 reivindica el principio de preferencia nacional y construye su argumentación sobre el término «nación» en base al «derecho a la diferencia»y a la propia cultura, donde los nacionales deben tener prioridad sobre los extranjeros en el acceso a los servicios públicos, sobre todo sanidad y educación. Estaríamos hablando de un nacionalismo propio de la derecha radical que se diferencia de otros movimientos nacionalistas que no poseen un discurso anti inmigración. Además, los últimos atentados yihadistas sucedidos en varios países de la Unión Europea desde el 7 de enero de 2015 en París han provocado una mayor centralización del discurso del odio hacia los musulmanes, la denominada «islamofobia» que promueve mensajes para generar actitudes de rechazo hacia este colectivo basadas en cuestiones culturales: «los seguidores del Islam son la mayor amenaza para la cultura europea y la identidad española» (España 2000, 2015) y también por cuestiones de seguridad nacional, al establecer como sinónimos los conceptos musulmán y terrorista. Esta práctica se extendió por parte de los partidos de extrema derecha tras los atentados acontecidos en Estados Unidos (11 de septiembre de 2001), en Madrid (11 de marzo de 2004), en Londres (11 de julio de 2005) y más recientemente tras los atentados de París, Bruselas y Niza en 2015 y 2016.

Otra de las variables centrales de su programa es el uso del término "pueblo», entendido como una comunidad nacional. De ahí su insistencia en definir legalmente quién pertenece a la nación y proponer estrictas medidas de control de la inmigración, el recorte de derechos de la población inmigrante ya instalada y la imposición del principio del ius sanguinis (derecho de sangre) para la obtención de la nacionalidad española, con el objetivo de limitar la concesión de la ciudadanía a los descendientes nacionales. El objeto final de estas medidas es la creación de un sistema legal de exclusión que pretende validar democráticamente y que responde a una concepción autoritaria del Estado.

Esta transformación ideológica ha estado acompañada por nuevas formas de activismo político que encuentran similitudes con las desarrolladas por otros partidos que han adoptado el mismo discurso, como Plataforma per Catalunya. Se trataría de una estrategia de arraigo, un «activismo comunitario y política de enraizamiento local» (HernándezCarr, 2012: 151), es decir, una actividad electoral centrada exclusivamente en el ámbito municipal, principalmente por la falta de recursos económicos y humanos, que permite el contacto directo con los vecinos más allá del trabajo institucional y que ha funcionado en los partidos 
socialmente etiquetados de extrema derecha que tratan de romper con este estigma político y público.

Mediante esta estrategia, el partido valenciano ha logrado ingresar en nuevos espacios públicos de ocio y recreo (en fiestas locales con la instalación de carpas como otras asociaciones y partidos) y además normalizar su presencia en la localidad donde implementan esta estrategia, ya que consiguen la participación de vecinos que no militan en el partido ni están adscritos a la extrema derecha tradicional, a través de temas que les afectan directamente. Esta técnica les confiere una imagen comprometida - frente al perfil distante y poco resolutivo- ofrecida por los partidos tradicionales y que se visibilizan en los barómetros de opinión que recogen el aumento de la desconfianza en los partidos políticos tradicionales, situándolos como uno de los principales problemas ${ }^{6}$ del país (CIS, 2017). En el caso de España 2000, la formación se centró en la denuncia de los múltiples casos de corrupción de los miembros del partido conservador mayoritario y principal rival político, el Partido Popular.

En el terreno institucional, la actividad del partido ha aumentado y se ha modificado en algunos aspectos. Desde 2003 hasta 2007 su concejal en la localidad de Silla apenas desarrolló un trabajo institucional destacado, concretamente presentó 5 mociones en 4 años sobre cuestiones que reivindicaban únicamente la identidad nacional. Sin embargo, a partir de 2007 y sobre todo desde 2015, con dos concejales, su actividad institucional no solo ha crecido sino que las temáticas se han transformado. Por ejemplo, el discurso de presentación en el primer Pleno del ayuntamiento de Silla celebrado el 13 de junio de 2015 es un claro indicador de la nueva retórica y las temáticas del discurso que está adoptando el partido. Lejos de los tradicionales mensajes de «iViva España!», «Nunca catalanes» o los más actuales «Frenemos el Islam», el concejal Andrés Vicent Zaragoza abogó por un trabajo continuado y próximo a los problemas de los vecinos de Silla, agradeciendo la labor de la militancia de la localidad. En este sentido, en la última legislatura (2015-actualidad) se aprecia una mayor actividad y participación en cada Pleno, aportando propuestas, presentando interpelaciones y preguntas relacionadas mayoritariamente con problemas que afectan directamente a los ciudadanos de la localidad (ruido, limpieza). También la reivindicación

6 El Eurobarómetro elaborado por el CIS de febrero de 2015 determinó que el $6,5 \%$ de los encuestados sitúa entre los principales problemas que existen actualmente en España a "Los/as políticos/as en general, los partidos y la política". Un año más tarde la cifra aumentó hasta el 9,7\% (CIS, 2016) y en febrero de 2017 volvió a crecer hasta situarse en el 10\% (CIS, 2017). 
de la identidad nacional está presente pero con el objetivo de cumplir con la legalidad vigente de la Constitución sobre la cuestión de símbolos e imágenes. La relación con las otras fuerzas políticas se ha traducido en la mayoría de los casos por la abstención y oposición a las mociones de los otros grupos políticos. En el caso de votar a favor, lo ha realizado no únicamente con el PP - que puede parecer su aliado político-ideológico más cercano-, sino con el resto de fuerzas que han presentado propuestas que afectan al bienestar, la transparencia y la economía del municipio, como la creación de una comisión de ciudadanos para realizar una auditoría de la deuda y cuentas municipales, pero también el reconocimiento del genocidio armenio.

Finalmente, el partido firmó el 23 de abril de 2016 un pacto con Plataforma per Catalunya y Plataforma por la Libertad y constituyeron la coalición RESPETO. El congreso se celebró en la población catalana de Coma-Ruga y eligió a José María Ruiz Puerta, presidente de Plataforma por la Libertad al frente de la dirección general de la federación, y Augusto Armengol y Rafael Ripoll, de Plataforma per Catalunya y España 2000 como vicepresidentes. A este congreso fundacional acudió Tatjana Festerling, la número dos de la organización islamófoba alemana Pegida (España 2000, 2016).

\section{Una discreta evolución electoral}

Tanto en las elecciones generales como en las europeas, España 2000 no ha obtenido representación, particularidad que comparte con otra formación de derecha radical populista española en el siglo XXI, Plataforma per Catalunya. No obstante, el partido no presentó candidatura ni en las elecciones europeas de $2014 \mathrm{ni}$ en las generales de diciembre de 2015. Para explicar esta última ausencia ante su militancia, España 2000 publicó un documento titulado «Ante las Elecciones Generales del 20 de diciembre de 2015" (España 2000, 2015). En él, ataca a los valores jurídicos de la Constitución porque consideran que no se respeta el derecho a la igualdad y el pluralismo político y animan a construir la alternativa que defienda la soberanía, identidad e independencia de los españoles. Sobre los nuevos partidos, manifiestan que "pretenden regenerar todo desde un mero reformismo con receta ultra liberal o bien con el reciclaje de las recetas marxistas» (España 2000, 2015).

El examen de la trayectoria electoral autonómica y municipal del partido desde 2003 hasta 2015 muestra una evolución creciente durante tres elecciones consecutivamente pero discreta, ya que su posición es 
secundaria e incluso marginal en la escena política valenciana (Tabla I). Así pues, el partido ha apostado por la política local como eje de su estrategia de crecimiento electoral (se han presentado prácticamente en todas las localidades de la Comunitat Valenciana desde 2007 hasta 2015). Entre otros motivos, por la falta de recursos económicos y de una organización excesivamente centralizada en la ciudad de Valencia.

En este sentido, el partido participó por primera vez en los comicios autonómicos de 2003 y obtuvo 2.650 votos, en 2007 la formación supera los 6.000 votos y consiguió dos concejales. Cuatro años más tarde (2011), la formación duplicó estos resultados (12.191) y extendió su número de concejales hasta cuatro. Además, desde 2007 y hasta 2015 el partido valenciano se ha posicionado como la primera fuerza de extrema derecha de la Comunitat Valenciana, frente a otras opciones de este espectro ideológico como Democracia Nacional, Falange Auténtica, Frente Nacional o Partido Social Patriota Español.

Las elecciones autonómicas celebradas en mayo de 2015 concluyeron con este ciclo alcista y, por primera vez, el partido redujo el número de votos (casi el 40\%) con una cifra menor a la obtenida en 2011. Igualmente su representación institucional se reduce a un solo concejal en la localidad de Silla (Valencia), a pesar de los esfuerzos de la formación en presentar candidatos en prácticamente el $99 \%$ de los municipios de la Comunitat Valenciana.

TABla 1. EVOLUCión EN LAS AUTONÓMiCAS Y LOCALES 2003-2015 DE ESPAÑA 2000

\begin{tabular}{lcc}
\cline { 2 - 3 } 2003 & Elecciones & Votos \\
2003 & Locales & 998 \\
2004 & Autonómicas & 2.650 \\
2007 & Legislativas & 2.721 \\
2007 & Locales & 3.790 \\
2008 & Autonómicas & 5.934 \\
2011 & Legislativas & 3.875 \\
2011 & Locales & 8.066 \\
2015 & Autonómicas & 12.191 \\
\hline 2015 & Locales & 5.591 \\
\hline
\end{tabular}

Fuente: elaboración propia con datos del Ministerio de Interior y Argos 
Atendiendo a la distribución territorial de los votos recibidos por la formación, se identifica un doble patrón en todas sus citas electorales (autonómicas y locales): una concentración de votos en la ciudad de Valencia y en las localidades y comarcas más próximas a su área metropolitana, caracterizadas por tener un tamaño mediano e incluso grande (80.000 habitantes). Por el contrario, en las provincias de Castellón y Alicante, el partido crece en municipios pequeños y comarcas alejadas de la capital.

Esta importante pérdida de votos supuso una decepción para el partido, especialmente en los ayuntamientos con concejales en Silla, Onda, Dos Aguas y en la ciudad de Valencia donde la acción social y de protesta ha sido más activa a lo largo de estos últimos cuatros años (2011-2015). Esta actitud activa ha consistido en el contacto directo con los vecinos mediante la intervención en diversas problemáticas vecinales asociadas a la inmigración, la corrupción y la reivindicación de la identidad nacional (reparto de alimentos solo para nacionales, manifestaciones en contra de la construcción de mezquitas, actos protesta en los juzgados de Valencia por asuntos de corrupción o la denuncia de un supuesto abuso de los espacios públicos por parte de otras religiones). Igualmente el partido ha mantenido este contacto directo con los vecinos en espacios de ocio y recreo en los municipios donde tienen sedes (durante las fiestas populares con casetas propias o cenas a lo largo del año).

\section{EL PERFIL DEL VOTANTE RADICAL}

En cuanto al perfil específico del votante, existe un mayor consenso entre los expertos que han abordado el tema, especialmente a través del análisis de las encuestas que permiten describir con mayor precisión el perfil sociodemográfico del electorado radical. Se trata de un electorado «heterogéneo en cuanto a sus características sociodemográficas» (Mayer y Perrineau, 1992) y sobrerrepresentado por «individuos de estratos sociales bajos y bajos niveles de educación, trabajadores manuales de baja calificación y parados» (Antón Mellón y Hernández Carr, 2016: 18).

En el caso de España 2000, al no existir encuestas que permitan la observación de actitudes, opiniones y características sociodemográficas que son imposibles de obtener a través de resultados electorales oficiales, se ha elaborado un pequeño estudio que examina el perfil de los individuos auto ubicados en el eje político 9-10, correspondiente a la extrema derecha. 
Se ha considerado que el votante de España 2000 se auto posiciona mayoritariamente en este espacio del continuum ideológico siguiendo la hipótesis más unánime en la literatura académica sobre la extrema derecha española (Pardos-Prado, 2012; Casals Meseguer, 2013; Alonso y Rovira, 2015). Sus trabajos coinciden en que el votante mayoritario procede al partido conservador, el Partido Popular, y a diferencia de otros partidos de extrema derecha como el Frente Nacional o Plataforma per Catalunya, el trasvase de votos no proviene de votantes identificados en espacios tradicionales de izquierda o ciudadanos desvinculados de la política (Mayer, 1992; Quilian, 1995; Pardos-Prado, 2012).

Para elaborar este estudio, se comparan diferentes variables (sociodemográficas, socioeconómicas y políticas) de la pregunta sobre el auto posicionamiento ideológico: "Cuando se habla de política se utilizan normalmente las expresiones izquierda y derecha. ¿En qué casilla se colocaría Ud.?» (encuestas postelectorales de las elecciones autonómicas del CIS 2003, 2007, 2011 y 2015). Las respuestas se han recodificado considerando los valores de 1 a 2 como extrema izquierda, 3 a 5 como izquierda, 6 a 8 como derecha y de 9 a 10 de extrema derecha.

El análisis revela que el perfil mayoritario de sujetos que se identifican con las posiciones de extrema derecha en los comicios de 2003 y 2007 son varones, adultos (especialmente de 55 a 65 años), sin estudios y obreros. Sin embargo, en 2011 y en plena crisis económica, este perfil se modifica sustancialmente y por primera vez prevalecen las mujeres y aumentan los porcentajes de jóvenes (18 a 30 años) con estudios universitarios. Este grupo es el que más ha crecido en tres citas electorales (2003-2011), aunque continua siendo minoritario frente a los jubilados.

No obstante, la tendencia desde 2011 hasta 2015 es la configuración de un electorado donde predominan dos grupos de edad opuestos: jubilados y jóvenes.

Por último, la variable «recuerdo de voto» revela que el voto no procede de sujetos del espacio de izquierdas o de la abstención, sino que es un voto mayoritariamente conservador, es decir, ubicado en el espacio de la derecha que tanto en la Comunitat Valenciana como en España corresponden al Partido Popular (hasta 2015). Esta hipótesis coincide con que el voto a las formaciones de extrema derecha españoles tiene un origen en el Partido Popular (Casals Messeguer, 2013 y Alonso y Rovira, 2015).

De manera muy reducida y únicamente en 2011 aparece un porcentaje de «nuevos votantes». Coincide con que es el año en el que obtienen los mejores resultados. Son prácticamente inexistentes los perfiles ideológicos comunistas o poscomunistas a lo largo de su historia. 
El resultado en su conjunto muestra que la formación valenciana es «atípica» respecto a las formaciones neopopulistas europeas, al menos en la cuestión del origen ideológico de su electorado, ya que no se ha producido una conversión de votantes ubicados en el espacio de la izquierda. En el caso valenciano sucede lo contrario, proceden del voto conservador y de nuevos votantes.

Sin embargo, hemos detectado dos similitudes importantes con los partidos radicales europeos de extrema derecha: el grupo de nuevos votantes contemplado en la encuesta de 2011 se identifica con el votante de Plataforma per Catalunya y son «jóvenes que se incorporan por primera vez a la vida política, que lógicamente no han podido votar antes y tampoco se pueden considerar antiguos votantes abstencionistas» (Pardos- Prado, 2012: 188).

Sobre este grupo de edad, esta investigación plantea que uno de los posibles factores que ha influido a la hora de captar su voto es la intensa campaña de España 2000 realizada a través de internet y de las redes sociales, y que se ha intensificado desde 2014 mediante mensajes que desde la Organización para la Seguridad y Cooperación Europea (OSCE) en 2003 ya calificó como «discursos del odio» (OSCE, 2003). Se trata de contenidos que difunden el odio hacia los musulmanes y a los inmigrantes, sobre todo, tras los diversos atentados yihadistas sucedidos desde enero de 2015 en París y en el resto de Europa. A través de este nuevo vehículo de comunicación, que es el más utilizado por los jóvenes desde 2008 (Fundación Orange, 2016: 61) y está caracterizado por el anonimato, impunidad, inmediatez e interconexión global, los partidos y organizaciones de extrema derecha han conseguido romper el monopolio en la oferta informativa asumida por los medios de comunicación convencionales (Oleaque, 2015).

En segundo lugar, respecto al perfil sociodemográfico, parece identificarse con una parte del electorado de España 2000 que se mantiene a lo largo de cuatro elecciones consecutivas, donde prevalecen los sujetos sin estudios o con estudios básicos, obreros no cualificados y cualificados (Antón Mellón y Hernández Carr, 2016). 


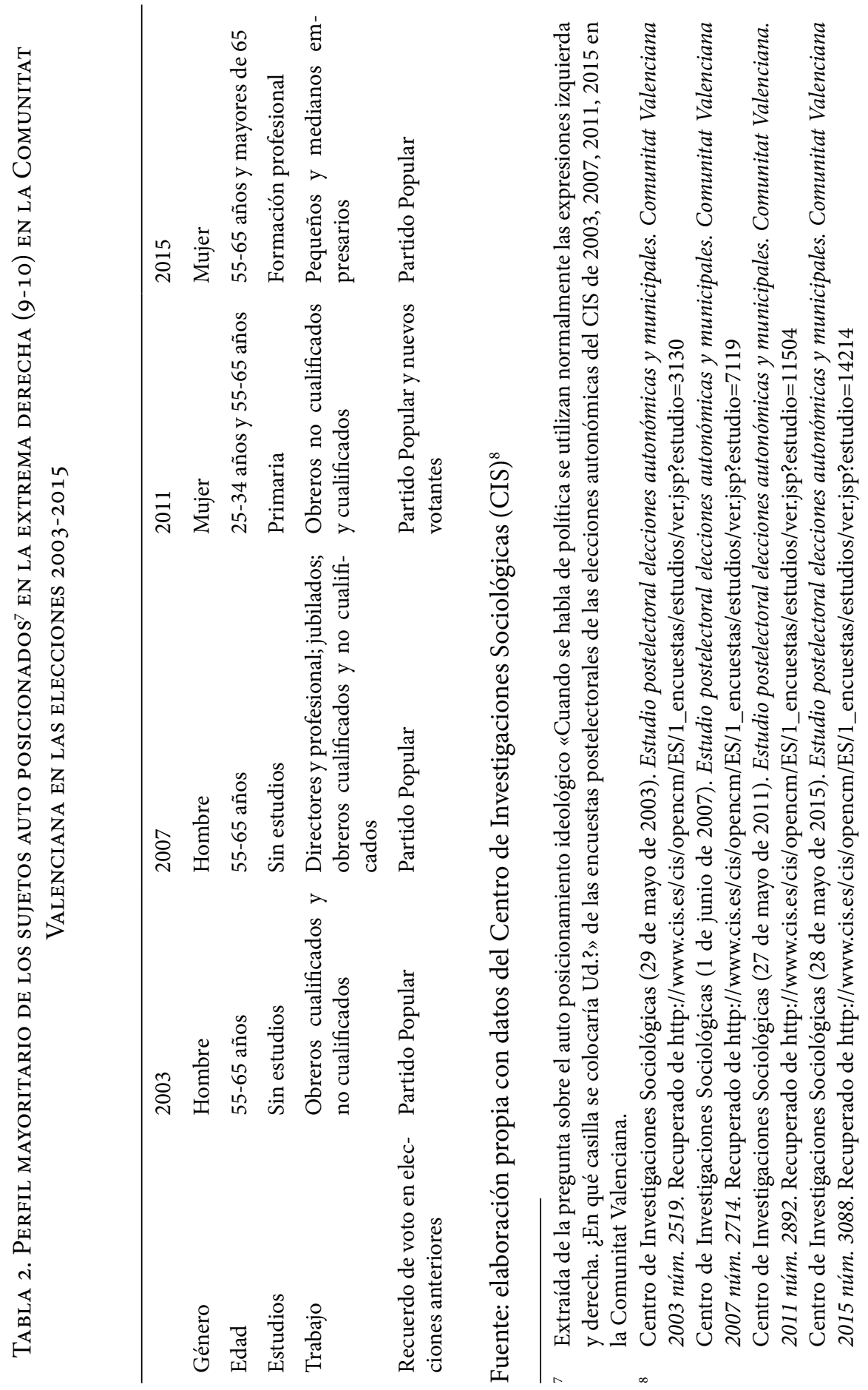




\section{FACTORES QUE HAN PROPICIADO LA EMERGENCIA RADICAL}

En primer lugar, la literatura académica ha argumentado de manera unánime que en España existe una demanda electoral de propuestas propias de la «derecha radical populista». Así, Alonso y Rovira (2015) y Hernández-Carr (2012) coinciden en señalar dos cambios fundamentales que explicarían esta situación: se ha producido un incremento de las actitudes y sentimientos anti inmigrantes en España como resultado de la llegada masiva de inmigrantes desde finales de la década de los noventa. En segundo término, ha crecido la falta de confianza en las instituciones democráticas, y una preferencia por cuestiones de ley y orden debido a una creciente preocupación por la seguridad. En el caso valenciano, el Informe Raxen (2016) y los Informes de delitos de odio elaborados por el Ministerio del Interior desde 2014 han constatado el incremento de actitudes y actos racistas y xenófobos en estos últimos años en el conjunto del Estado español. En concreto, la Comunitat Valenciana es junto con Madrid la autonomía donde se producen más delitos de esta categoría desde 2014 hasta 2016.

Al mismo tiempo, la Comunitat Valenciana se ha convertido desde el año 2000 en una de las regiones europeas con mayor tasa de población inmigrante del Estado español junto con Baleares, Madrid y Cataluña. El Instituto Nacional de Estadística (INE) registró una tasa de inmigración en el año 2002 del 2,3\%, y diez años más tarde, del 12,2\% (en el año 2017 se encuentra en el 9\%). Durante estos años, la Comunitat Valenciana ha sido - junto con Baleares, Madrid, Barcelona - una de las comunidades con más tasa de inmigración del Estado español.

De esta manera, y partiendo de este contexto que confirma la existencia de una demanda electoral, se ha planteado el estudio de los factores que han propiciado la emergencia de España 2000 en la Comunitat Valenciana como partido político que adopta un discurso propio de la «derecha radical populista», ya que «España 2000 es el equivalente a Plataforma per Catalunya en el País Valencià» (Prados-Prado, 2012: 68). La formación catalana se convirtió en el primer partido durante el periodo democrático que adoptó un discurso neopopulista y con el que consiguió mantener durante varias elecciones autonómicas una importante representación institucional (Hernández Carr, 2012).

Sin embargo, debido a la inexistencia de encuestas individuales o agregadas que permitan cuantificar la transferencia de voto a España 2000, como sucede en el caso de otras formaciones radicales con una mayor trayectoria nacional o internacional (Frente Nacional, Partido por la Libertad, Auténticos Finlandeses) y así poder analizar el origen 
del electorado del partido, hemos recurrido al grupo de teorías que proponen una «estructura de oportunidades» (Arzheimer, 2009) necesarias para que emerjan esta clase de formaciones. En concreto, intentamos explicar la emergencia del fenómeno valenciano a través de la teoría diseñada por Jackman y Volpert (1996).

De esta manera, hemos analizado factores relativos al contexto político y social e institucional en la Comunitat Valenciana que han podido beneficiar o perjudicar la emergencia y posible consolidación de España 2000.

Estos autores proponen tres hipótesis que favorecerían la emergencia y crecimiento de estos partidos: 1) la extrema derecha crece a medida que aumenta el paro en un determinado país, 2) cuando hay más techo en un determinado sistema de partidos, más reducidas son las expectativas electorales de la extrema derecha, 3) la combinación del multipartidismo con un sistema electoral proporcional está asociada a un apoyo alto a la extrema derecha.

En cuanto a la primera hipótesis de Jackman y Volper, nuestro análisis muestra una coincidencia entre municipios que reciben más votos -y que están ubicados en las comarcas más próximas a la ciudad de Valencia - con lo que tienen mayores tasas de paro de la Comunitat, o al menos con porcentajes superiores a la media de la autonomía y especialmente en las elecciones de 2011. Además, en el examen de la tasa de paro y los intervalos de población más afectados destaca que desde 2003 la franja de población con más tasa de paro son los menores de 25. Precisamente, una parte importante de militantes de España 2000, según el seguimiento de los actos en campaña y las diferentes entrevistas, así como el estudio propio con las encuestas del CIS, coinciden con este perfil de población.

En segundo lugar, los autores aseguran que "cuando hay más techo en un determinado sistema de partidos, más reducidas son las expectativas electorales de la extrema derechas». Sobre la cuestión de la proporcionalidad del sistema electoral valenciano, el Estatuto de Autonomía (arts. 23 y 24) y su concreción en el art.12 de la Ley 1/1987 Electoral Valenciana, del 31 de marzo, fijan la barrera electoral del 5\%. Esta ha sido objeto de crítica desde ámbitos partidistas (Partit Socialista del País Valencià, Esquerra Unida, Bloc, Ciudadanos) y académicos por la desproporcionalidad territorial que provoca.

«La disfunción fundamental, clara y evidente es, por sus efectos directos, la exclusión de la representación en las Cortes de fuerzas políticas relevantes como consecuencia de la aplicación de la barrera electoral del 5\% del conjunto de los votos emitidos en la Comunitat 
Valenciana (...) parece poco razonable que, en el contexto electoral valenciano donde el partido más votado obtiene poco más de un millón de votos, se excluya de la representación a fuerzas políticas con más de 100.000 votos o, dicho de otra forma, que 100.000 valencianos que apoyan un proyecto político determinado no puedan hacer oír su voz en el parlamento.» (Martín Cubas, 2016: 102).

En la última hipótesis, los autores plantean el hecho de que la extrema derecha se desarrollará con mayor facilidad en los países donde exista un sistema proporcional y multipartidista donde la extrema derecha puede captar «espacios políticos» desatendidos.

En el caso de España 2000 en la Comunitat Valenciana tenemos que, por una parte, el sistema político no fomenta la pluralidad y tampoco la entrada de partidos minoritarios, como es España 2000, ya que la barrera electoral es del 5\%. Por lo tanto, es más complicado que se produzca una fragmentación del sistema de partidos. Por otro lado, sin embargo, el espectro político, sobre todo del lado de la derecha, no está tan fragmentado como en otros países (Italia, Francia, Holanda, Austria) y reduce las posibilidades de encontrar un "espacio desatendido" por parte de España 2000. Este hecho conecta directamente con el fracaso de la extrema derecha española y explicaría por qué España 2000 no alcanzaría tan buenos resultados como otros partidos radicales europeos. El principal partido conservador, el Partido Popular, aglutina los votos de todo el espacio político de la derecha, incluido los radicales (Casals Messeguer, 2004).

En este sentido, desde 1999 hasta 2015, el Parlamento Valenciano se ha caracterizado por un bipartidismo en el que el Partido Popular tenía mayoría absoluta, y por tanto, era la fuerza hegemónica del espacio electoral de la derecha. Con la irrupción de España 2000 en 2003 y especialmente hasta 2011, planteamos que aunque el Parlamento Valenciano no se encuentre fragmentado, la formación radical ha logrado quebrantar - de manera mínima- en el espacio político de la derecha y, en concreto, del Partido Popular. El resultado sería un votante de nueva extrema derecha en la Comunitat Valenciana, diferenciado del Partido Popular, que se ha movilizado por la propuesta anti inmigración de España 2000 por su carácter radical e incluso racista. En este sentido, y siguiendo la terminología de Jackman y Volper, España 2000 ha logrado encontrar un espacio político que ha sido «desatendido» por el Partido Popular y que identificamos con la inmigración. De hecho, el Partido Popular en la Comunitat Valenciana aprobó en 2008 la Ley 15/2008 de Integración de las Personas Inmigrantes en la Comunitat Valenciana, del 5 de diciembre, de carácter progresista, que fue objetivo principal de críticas y descali- 
ficaciones desde España 2000 en esa campaña electoral por la vocación integradora de la Ley.

\section{Conclusiones}

El artículo ha analizado la evolución política y electoral de España 2000 desde las elecciones autonómicas y municipales de 2003 hasta las de 2015. Durante sus más de diez años de historia, el partido ha transformado su discurso y principales estrategias propagandísticas y ha incorporado elementos similares a las de otras formaciones neopopulistas europeas definidas como «derecha radical populista» o «nueva extrema derecha».

La investigación ha distinguido dos etapas donde se han evidenciado estas transformaciones: la primera, desde 2003 hasta 2007, los ejes de movilización del partido se centraron en la exaltación de la unidad española y el anti catalanismo y su actividad política se focalizaba en manifestaciones y actos protesta.

En un segundo periodo (2007-2015), y con motivo de las elecciones autonómicas y municipales en la Comunitat Valenciana, la formación adoptó una nueva estrategia que se concentró en tres aspectos: un nuevo discurso centrado en el eje autóctonos-extranjeros (anti inmigración) y se visibilizó en acciones como el reparto de alimentos solo para nacionales o manifestaciones en contra del Islam; la nueva imagen de algunos de sus líderes (más jóvenes y formados); la adopción de una línea de trabajo basada en el "activismo comunitario», es decir, una actividad electoral centrada exclusivamente en el ámbito municipal como realizó Plataforma per Catalunya en 2010 y 2011 y que le reportó los mejores resultados electores de su historia.

Pero, ¿qué factores han contribuido a la emergencia de este partido? El artículo ha recurrido al grupo de teorías que proponen una «estructura de oportunidades políticas» (Arzheimer, 2009) que explican la aparición y persistencia de la extrema derecha en un determinado territorio. En este sentido, hemos partido de la consideración unánime en la literatura académica de que en España ya es posible hablar de una demanda hacia este tipo de partidos, entre otros motivos por el debilitamiento de la identificación partidista, un descenso en la valoración que los electores hacen de los partidos tradicionales y el fenómeno de la inmigración que se ha consolidado como una temática distinguida en la esfera social y política (Hernández-Carr, 2012; Alonso y Rovira, 2015). 
Otro de los motivos de la elección de esta teoría, en concreto la planteada por Jackman y Volper (1996), se debe la inexistencia de encuestas sobre la trasferencia de voto o actitudes de su electorado de nuestro objeto de estudio. En este sentido, la aplicación de esta teoría ha permitido detectar factores como el número de inmigrantes, el índice de parados o el diseño institucional, similares a otras formaciones neopopulistas europeas que explicarían la emergencia de España 2000.

En primer lugar, se ha evidenciado una correlación entre los municipios que tienen mayores tasas de desempleo e inmigración y los que más votos de España 2000 han recibido e incluso donde más han crecido a lo largo de su ciclo electoral. En segundo lugar, como también sucede con otras formaciones europeas, la existencia de un sistema electoral desproporcional no ha beneficiado al partido, ya que el sistema electoral valenciano se caracteriza por tener una barrera electoral del $5 \%$ que no fomenta la pluralidad ni tampoco la entrada de partidos minoritarios. Finalmente, la fragmentación política solo ha favorecido a España 2000 cuando se ha producido una mínima segmentación en el espacio político de la derecha y no en todo el continnum político (en 2015, el Parlamento Valenciano es el más fragmentado de su historia y el partido pierde el $40 \%$ de sus apoyos). En este sentido, la irrupción de España 2000 se ha producido cuando el partido ha sido capaz de encontrar un espacio desatendido, que en nuestro análisis determinamos que es el vinculado con la inmigración y la adopción de un discurso restrictivo e incluso racista con el que se ha diferenciado de la opción mayoritaria conservadora que, como partido en el gobierno, ha aprobado medidas de carácter integrador e incluso progresista. No obstante, los resultados obtenidos de los análisis de su electorado evidencian que, aunque es cierto que España 2000 crece por los votantes del Partido Popular, una parte muy elevada de su voto, al menos a nivel comarcal y local, parece proceder del grupo de nuevos votantes, abstencionistas.

Al mismo tiempo, la investigación del perfil sociodemográfico del posible votante de España 2000 muestra que es heterogéneo en cuanto a la edad, sexo, nivel de estudios y profesión, y que además se ha transformado a lo largo de su ciclo electoral. En concreto, el examen en profundidad revela que el perfil mayoritario que se repite a lo largo de las dos últimas elecciones autonómicas (2011-2015) se divide en dos grupos de edad muy antagónicos: jubilados y jóvenes, los segundos posiblemente han aumento durante este espacio de tiempo por la intensa campaña realizada a través de internet y de las redes sociales. Asimismo, el electorado de la formación valenciana tiene elementos en común con el de los partidos europeos radicales, sobre todo en lo referente a otras 
características sociodemográficas (bajo nivel de estudios, obreros y clase baja), pero a diferencia de las formaciones europeas, sus votantes son mayoritariamente del partido conservador y no se identifican con posiciones de izquierda.

Este origen conservador del electorado de España 2000 (junto con los nuevos votantes y abstencionistas) supone uno de los motivos que explica que el proyecto político valenciano no haya conseguido crecer electoralmente como otras formaciones europeas que han adoptado un discurso similar, pero este factor no es el único. Este estudio propone que se debe a la interrelación de elementos internos y externos al proyecto político.

Por un lado, y vinculado a este origen conservador del votante de España 2000, en España y en la Comunitat Valenciana, el Partido Popular actúa como una especie de partido atrapalotodo en el espacio político de la derecha. Al mismo tiempo, y en parte explicaría esta capacidad de atracción del voto del Partido Popular, la estrategia de este ha incorporado en algunas ocasiones demandas de los partidos de extrema derecha (Alonso y Rovira, 2015). La estructura de eje del sistema político español se subdivide en uno socio económico (derecha vs. izquierda) y otro nacionalista (centro vs. periferia) y es precisamente este último factor (centro vs. periferia) el que ha obstaculizado el auge de la extrema derecha, ya que a los partidos de extrema derecha les resulta prácticamente imposible jugar la carta nativista (Alonso y Rovira, 2015).

Por otro lado, los principales motivos que han impedido un crecimiento continuado y más acusado de España 2000 se deben a elementos internos de la propia formación y que se han convertido en "crónicos»: la figura del líder del partido, José Luis Roberto, y de algunos de sus portavoces vinculados con el fascismo y la violencia y sin apenas carisma; la falta de una organización estructurada, sólida y con suficientes recursos económicos en toda la Comunitat Valenciana que no se reduzca a la provincia de Valencia; y la excesiva centralidad del discurso anti inmigración que le origina una fuerte dependencia del tipo de política migratoria (progresista o más radical) que adopte el Partido Popular, ya que hasta el momento es una de sus principales fuente de votos.

Respecto a su posible irrupción en el parlamento nacional, los resultados de las elecciones autonómicas de 2015 dejaban a España 2000 muy lejos de poder ser una formación de implantación nacional y con capacidad de incorporarse a la primera línea de la política valenciana. Sin embargo, la alianza de España 2000 con otras formaciones españolas de extrema derecha formalizada en abril de 2016 con el nombre Respeto, será determinante para la extensión del partido a nivel nacional y 
europeo. Esta alianza cuenta con Plataforma per Catalunya ubicada en Cataluña y Plataforma por la Libertad en Madrid. Por este motivo, la experiencia de la formación catalana puede ser fundamental para construir una nueva oferta política de carácter radical populista similar a las europeas para las elecciones nacionales en 2020.

\section{REFERENCIAS}

Alonso, S. y Rovira Kaltwasser, C. (2015). Spain: No Country for the Populist Radical Right? South European Society and Politic, 20(1), 1-21.

Antón-Mellón, J. y Hernández-Carr (2016). El crecimiento electoral de la derecha radical populista en Europa: parámetros ideológicos y motivaciones sociales. Política y Sociedad, 53(1), 17-28.

Arzheimer, K. (2009). Contextual Factors and the Extreme Right Vote in Western Europe, 1980-2002. American Journal of Political Science, 14(2), 259-275. Ayuntamiento de Silla. (30 de enero de 2015). Acta del pleno municipal. Recuperado de http://www.valencia.es/ayuntamiento/ plenos/A_00001_20150130_1045_0_firmadoweb.pdf

Betz, H-G. (2004). La droite populiste en Europe. Extrême et démocrate? Paris: Autrement.

Birenbaum, G. (1992). Le Front national en politique. Paris: Balland.

Bommes, M. y Geddes, A. (2000). Immigration and Welfare. Challenging the Borders of the Welfare State. Londres: Routledge.

Breivick, A. (2011). Una declaración de independencia europea 2083. Recuperado de http://www.ssb.no/a/publikasjoner/pdf/notat_200633/notat_200633.pdf

Camus, J. (2015). Les Droites extrêmes en Europa. Ciudad de impresión: Seuil.

Casals, X. (1995). Los neonazis en España. Madrid: Grijalbo.

Casals, X. (2003). Ultrapatriotas. Extrema derecha y nacionalismo de la Guerra Fría a la era de la globalización. Barcelona: Crítica.

Casals, X. (2011). La nova dreta populista i l'"enigma espanyol". L'Espill, 38(1), 82-91. Centro de Investigaciones Sociológicas. (2003). Estudio postelectoral elecciones autonómicas y municipales. Comunitat Valenciana 2003 núm. 2693. Madrid: Autor.

Centro de Investigaciones Sociológicas. (2007). Estudio postelectoral elecciones autonómicas y municipales. Comunitat Valenciana 2007 núm. 2714. Madrid: Autor. 
Centro de Investigaciones Sociológicas. (2011). Estudio postelectoral elecciones autonómicas y municipales. Comunitat Valenciana. 2011 núm. 2892. Madrid: Autor.

Centro de Investigaciones Sociológicas (2015). Estudio postelectoral elecciones autonómicas y municipales. Comunitat Valenciana 2015 núm. 3088. Madrid: Autor.

De Lange, S. y Mudde, C. (2005). Political Extremism in Europe. European Political Science, 4(2), 476-88. El TS desestima el recurso del Bloc contra la barrera electoral del 5\% de las elecciones de 2007 (17 de noviembre de 2011). Europa Press. Recuperado de http://www.europapress.es/comunitat-valenciana/noticia-ts-desestima-recurso-bloc-contra-barreraelectoral-elecciones-2007-20111117190042.html

España 2000 (2014). En el Manifiesto del partido ante las elecciones europeas de mayo 2014. Madrid: Autor. Recuperado de http://espana2000. org/?page_id=43

España 2000 (2015). Ante las Elecciones Generales del 20 de diciembre de 2015. Madrid: Autor. Recuperado de http://espana2000.org/?p=3601

España 2000 (7 de febrero de 2016). Se preparan las bases para conformar la alternativa patriótica en España. Madrid: Autor. Recuperado de http:// espana2000.org/?p=3729

España 2000 (12 de mayo de 2017). Manifiesto de España2000 ante las próximas Elecciones Europeas. Madrid: Autor. Recuperado de http://espana2000.org/?p=2454\#more-245

Evans, J. (2005). The Dynamics of Social Change in Radical Right-Wing Populist Party Support. Comparative European Politics, 3(1), 76-101. Flor, V. (2011). Noves glòries a Espanya. Anticatalanisme $i$ identitat valenciana. Barcelona: Afers.

Fundación Orange. (2016). Informe anual sobre el desarrollo de la sociedad de información en España. Madrid: Fundación Orange.

Gallego, F. (2008). Nostalgia y modernización. La extrema derecha española entre la crisis final del franquismo y la consolidación de la democracia (1973-1986). Historia contemporánea, 7(2), 245-265. Ciudad: Editorial.

Gil, J. (2012). Ultras con piel de ONG. El País. Recuperado de http://ccaa. elpais.com/ccaa/2012/11/17/valencia/1353176811_374796.html

Givens, T. (2004). The Radical Right Gender Gap. Comparative Political Studies, 37(1), 30-54. Ciudad: Editorial.

Goodwin, M. (2007). Grandpa's Fascism and the New Kids on the block: Contemporary Approaches to the Dark Side of Europe. Ethnopolitics, 6(1), 145-54. Ciudad: Editorial. 
Griffin, R. (2000). Interregnum or Endgame? Radical Right Thought in the 'Post-fascist' era. The Journal of Political Ideologies, 5(2), 163-78. Ciudad: Editorial.

Hernández-Carr, A. (2012). La irrupción de la nueva extrema derecha en España: un análisis de la trayectoria, estrategia política y base electoral de Plataforma per Catalunya (Tesis doctoral). Universitat Autònoma de Barcelona, Bellaterra, Barcelona. Recuperado de http://www.tesisenred. net/handle/10803/96408

Ignazi, P. (2003). Extreme right parties in Western Europe. Oxford: Oxford University Press.

Jackman, R. y Volpert, K. (1996). Conditions Favoring Parties of the Extreme Right in Western. Europe. British Journal of Political Science, 26(1), 501521. Ciudad: Editorial.

La ultraderecha boicotea la presentación de un libro sobre el anticatalanismo en Valencia (5 de julio de 2011). El Mundo. Recuperado de http://www. elmundo.es/elmundo/2011/07/05/valencia/1309891348.html

López, A. (2016). La estrategia de la nueva extrema derecha en Internet. Revista electrónica de recursos en Internet sobre geografía y ciencias sociales, 41(1), 1-23.

Lubbers, M., Gijsberts, M. y Scheepers, P. (2002). Extreme right-wing voting in Western Europe. European Journal of Political Research, 31(1), 345-78.

Martín, J. (2016). El estatuto de autonomía como marco normativo de una reforma del sistema electoral de Les Corts Valencianes. Revista valenciana de reformes democràtiques, 1(1), 99-129.

Mayer, N. y Perrineau, P. (1992). Why do they Vote for the Le Pen. European Journal of Political Research, 22(1), 123-141.

Ministerio del Interior de España. (2014). Informe sobre incidentes relacionados con los delitos de odio en España. Madrid: Autor.

Ministerio del Interior de España. (2015). Informe sobre incidentes relacionados con los delitos de odio en España. Madrid: Autor.

Ministerio del Interior de España. (2016). Informe sobre incidentes relacionados con los delitos de odio en España. Madrid: Autor.

Movimiento contra la Intolerancia. (2014). Informe especial Raxen. Europa en crisis: tolerancia o barbarie: racismo, xenofobia e intolerancia en internet. Madrid: Autor.

Mudde, C. (2007). Populist Radical Right Parties in Europe. Cambridge: Cambridge University Press.

Oleaque, J. (2015). Racismo en internet: webs, redes sociales y crecimiento internacional. Valencia: Universidad Internacional de Valencia. 
Organización para la Seguridad y Cooperación Europea. (2003). Hate Crimes in the OSCE Región: Incidents and Responses. Polonia: Organización Interamericana de Desarrollo Humano (OIDDH).

Paniagua Fuentes, J. y Piqueras, J. A. (2005). Diccionario biográfico de políticos valencianos, 1810-2005. Valencia: Institut Alfons el Magnànim.

Pardos-Prado, S. (2012). Xenofóbia a les urnes. Sobre com la reacció contra els immigrants es transforma en resultats electorals. Barcelona: Larquer.

Periodistas obligados a recibir al líder neonazi. (7 de mayo de 2012). Diario vasco. Recuperado de http://www.diariovasco.com/videos/internacional/noticias-de-internacional/1625130352001-periodistas-obligados-recibir-lider-neonazis-griegos.html

Respeto. (1 de junio de 2016). La Federación Respeto recibe el apoyo de la líder Tatjana Festerling (Pegida). Respeto. Recuperado de http://www. respeto.eu/index.php/2016/06/01/congreso-fundacional/

Rius, X. (2012). Mapa incompleto de la extrema derecha. Recuperado de http://xavier-rius.blogspot.com.es/2012/08/mapa-incompleto-de-laextrema-derecha-2.html

Rodríguez, J. L. (2006). De la vieja a la nueva extrema derecha (pasando por la fascinación por el fascismo). Historia actual online, 9(2), 87-99. Recuperado de http://www.historiaactual.com/hao/pbhaoabs.asp?idi=ESP\& $\mathrm{pgt}=2 \& \mathrm{pid}=4 \& \mathrm{pbl}=\mathrm{HAO} \& \mathrm{vol}=1$ \&iss $=9 \& \mathrm{cont}=9$

Rydgren, J. (2005). Is Extreme Right-Wing Populism Contagious? Explaining the Emergence of a New Party Family. European Journal of Political Research, 44(1), 413-37.

Rydgren, J. (2007). The sociology of the radical right. Annual Review of Sociology, 3(1), 241-62.

Salas, A. (2004). El año que trafiqué con mujeres. Barcelona: Planeta.

Serrano Valencia, Á. (13 de octubre de 2012). El amanecer dorado de los ultras reúne a 200 personas. Levante. El mercantil valenciano. Recuperado de http://www.levante-emv.com/comunitat-valenciana/2012/10/13/ amanecer-dorado-ultras-reune-200-personas/943658.html

Taguieff, P-A. (2007). Interpretar la ola populista en la Europa contemporánea: entre resurgencia y emergencia. En Simón, M. A. (Ed.), La extrema derecha en Europa desde 1945 a nuestros días (pp. 39-66). Madrid: Tecnos.

Vázquez Silla, M. (1 de junio de 2011). Un edil de E2000 imputado en la operación Panzer. Levante. El mercantil valenciano. Recuperado de http:// www.levante-emv.com/comarcas/2011/06/01/edil-espana-2000-sillaimputado-operacion-extrema-derecha/811855.html

España 2000 (s/f). Reconstrucción Nacional. Madrid: Autor. Recuperado de http://espana2000.org/?page_id=41 